\title{
Optimal Routing Path Selection to Improve Data Transmission and Lifetime of Wireless Sensor Network
}

\author{
${ }^{* 1}$ Sumedha Sirsikar, ${ }^{2}$ Abhishek Chunawale \\ ${ }^{1,2}$ Department of Information Technology, Maharashtra Institute of Technology, Pune, India \\ Email:sumedha.sirsikar@mitpune.edu.in,abhishek.chunawale@mitpune.edu.in
}

Received: $20^{\text {th }}$ September2018, Accepted: $11^{\text {th }}$ October 2018, Published: $31^{\text {st }}$ October 2018

\begin{abstract}
The self-organizational ability of ad-hoc Wireless Sensor Networks (WSN) have led them to be the most popular choice. Despite significant advancements in Wireless Sensor Networks, efficient energy consumption in the network remains one of the most important research challenges. Clustering sensor nodes and organizing them hierarchically have proven to be an effective method to provide better data aggregation and scalability for the sensor network while conserving limited energy. Topology control mechanisms promotes efficient energy consumption as it deals with the arrangement of the nodes. Topology acts as virtual backbone of the network, so a good topology control mechanism improves the overall network lifetime. There are various techniques for topology control such as Self-Organizing algorithms, Minimal Spanning Trees and Unit Disk Graphs which are taken as basis for the proposed algorithm. The proposed algorithm performs hierarchical clustering on the network in order establish optimal routing path from sensor nodes to sink node whilst conserving network resources.
\end{abstract}

Keywords

Optimal Routing Path, Wireless Sensor Network, Topology Control

\section{Introduction}

Wireless Sensor Network (WSN) consists of randomly distributed wireless sensor nodes which are a large number of small sized, battery powered sensor nodes that have limited in battery energy, CPU power and communication capabilities. It consists of the area populated with distributed autonomous devices equipped with sensors to measure physical or environmental constants, For Example: Temperature, Pressure, Vibration, Emissions and many others. WSN are suitable for a great variety of environments and conditions, what broadens the scale of their applications.

The applications for WSN involve tracking, monitoring, and controlling. WSN are mainly utilized for habitat monitoring, object tracking, nuclear reactor control, fire detection, traffic monitoring etc.

WSN have limited power supply and hardware capability hence judicious use of resources is the key for having an energy efficient and durable network. For the same, unnecessary use of battery is avoided by communicating only for short periods of time (short bursts of time) since both transmitting and receiving are equally costly. Nodes are meant to collect data and transmit their information across the network towards the desired location (sink node). Without the provision of efficient methodology to gather and send data, due to enormous redundant messages being flooding in the network, sensor nodes might drain out of battery quickly. As a result various techniques have been designed to improve battery lifetime of nodes and prevent flooding of messages in network. One majorly used technique is Clustering.

Clustering is a useful technique through which we can affect the network lifetime, scalability and load balancing. The main objective of clustering protocol is to increase the WSN efficiency by organizing the network nodes into smaller clusters and electing a Cluster Head $(\mathrm{CH})$ for each cluster. Sensor nodes in each cluster transmit their data to the respective $\mathrm{CH}$ and the $\mathrm{CH}$ aggregates data and forward them to a central Base Station (BS). Once the WSN has been divided into clusters, the communication between nodes can be either intra cluster or inter cluster. Intra-Cluster communication comprises the message exchanges between the participating nodes and the $\mathrm{CH}$. Inter-Cluster communication includes the transmission of messages between the $\mathrm{CHs}$ or between the $\mathrm{CH}$ and the BS. Since only the $\mathrm{CH}$ is transmitting information out of the cluster collisions are avoided between the sensors inside the cluster because they don't have to share the communication channel with the nodes present in other clusters. Many clustering protocols have been proposed in WSN in recent years they can be divided into two main groups namely, Distributed and Centralized protocols. In the first, each sensor decides whether it will become a $\mathrm{CH}$ or not based on some criterion. In the second, a central node like the BS compares between the different nodes to select the best cluster heads among them. 
The main concern of Clustering is selection of Cluster Heads, load it carries and number of links formed which affects the network lifetime. The proposed algorithm forms hierarchical topology and selects optimal cluster heads according to load factor. The proposed algorithm restricts the degree of each $\mathrm{CH}$ to a limit defined by user which determines the value of node/load factor as per desired in network.

It further forms optimal links thus reducing flooding and conserving energy. The number of links formed are equal to number of nodes thus reducing redundant links and helps in improving network lifetime.

The second section gives detailed description of major research work done in the domain of network topology control. It focuses on three popular techniques namely, Minimum Spanning Tree (MST), Self-Organizing Systems (SOS) and Unit Disk Graphs (UDG). This section compares various features of these algorithms on various parameters. The third sections describes the proposed algorithm and its working. The fourth section illustrates the results of various scenarios obtained by simulating the algorithm and comparing it with MST, SOS and UDG. This section also contains the graphical representation of the behavior shown by the system in various scenarios. The fifth section summarizes the research work.

\section{Related Work}

Three major topology control methods were taken as base for the development of required algorithm. These methodologies are: Minimum Spanning Tree, Unit Disk Graphs and Self Organizing Systems.

In MST each node ' $u$ ' collects its two-hop neighbor's information N2(u). Then each node ' $u$ ' computes the Euclidian Minimum Spanning Tree (N2(u)) of all nodes N2(u), including ' $u$ ' itself. For each Edge uv $\epsilon$ MST $(\mathrm{N} 2(\mathrm{u})$ ), node ' $u$ ' tells node ' $v$ ' about this directed edge. Thus the node u keeps an edge 'uv' if uv $\epsilon$ MST (N2(u)) or $\mathrm{vu} \in \operatorname{MST}(\mathrm{N} 2(\mathrm{v}))$. Finally, each node uses its two hop information to construct such structure [1].

MST algorithm is applied after any clustering algorithm is applied on the nodes and clustering of the network has already taken place. MST algorithm then applies topology control on two levels namely Intra-Cluster topology control and Inter-Cluster topology control. In Intra-Cluster topology control most cost effective links are scanned within a cluster and a local MST is formed which spans all the nodes of the cluster. In Inter-Cluster topology control algorithm a global MST is created spanning all the CHs present in the network. MST consumes energy within a factor if the optimum it has its own disadvantages such as: Network Segregation, Network failure maybe caused by failure of even a single link and static in nature.

UDG [2] uses the mathematical concept of unit disks. The unit disk around some point ' $P$ ' is the set of points whose distance from ' $\mathrm{P}$ ' is less than or equal to 1 .

$\mathrm{D} 1(\mathrm{P})=\{\mathrm{Q}:|\mathrm{P}-\mathrm{Q}|<=1\}$

UDG is the intersection graph of family of unit disks in the Euclidian plane. Each disk has one vertex as its center which is a sensor node in case of WSN [3]. If vertices of two disks are in range of each other, then an edge can be formed between them.

UDG is one of the simplest models of topology control, hence it is widely used in Broadcast Network.

UDG also has its own demerits: It does not take geographic and environmental factors under consideration, temporal variation including mobility is not reflected in the model hence the model is static in nature.

Self-Organization algorithm is nature inspired clustering based algorithm. It reduces both computation complexity and energy consumption of the whole ad-hoc network. This clustering is done by grouping nodes inside a certain transmission area. Each of these nodes play different roles, namely Leader, Gateway, Bridge and Member: Each of them is controlled by a designated leader agent. This leader agent is selected according to a weight that is composed by the residual energy and the number of neighbors [1]. Table 1 shows the features of MST, SOS and UDG measured based on various parameters.

\begin{tabular}{|l|l|l|l|}
\hline Parameters & Minimum Spanning Tree & Self -Organizing System & Unit Disk Graph \\
\hline Network Type & Static & Both Static and Dynamic & Static \\
\hline Number of Links & Least & Intermediate & Most \\
\hline Longevity of CH Role & $\begin{array}{l}\text { Relinquishes role after certain } \\
\text { threshold }\end{array}$ & $\begin{array}{l}\text { Sustains role by reducing } \\
\text { energy }\end{array}$ & $\begin{array}{l}\text { Relinquishes role after certain } \\
\text { threshold }\end{array}$ \\
\hline Flooding & Least & Intermediate & Most \\
\hline Network Reconfiguration & Difficult & Relatively Easy & Easy \\
\hline Localization & Not much & Available & Available \\
\hline Need of Control Message & Required & Not Required & Not Required \\
\hline
\end{tabular}

Table 1. Comparison between MST, SOS and UDG. 


\section{Proposed Algorithm}

In WSN resources being scarce it is mandatory to connect the whole network with minimalistic use of the resources and conserve resources to maximum extent possible. The most common approach taken for this problem is to build the network by creating clusters based upon the first layer of nodes near the sink.

Going down the lane with this common approach ensures saving the energy of the nodes that lie to the bottom of the hierarchy but creates an overuse for the upper part of the hierarchy. As upper part of the hierarchy usually suffers with burden of over-load, it has the chance of creating a segmentation in the network at a later stage due to overusage of resources. This creates an imbalance in the network by creating shortage of resources for the nodes of upper layer hierarchy and leaving the layer below them with plenty of resources, even when the segmentation occurs these resources cannot be put to use. So now to avoid this imbalance a new strategy called fast clustering has been introduced in further sections. Following assumptions have been considered in designing the strategy:

Under this model there are some assumptions done that are listed as follows:

- Every agent has a unique identifier ID e.g., its IP address;

- Every agent knows their neighbors to one-hop;

- The agent can use overhearing to obtain important information for reducing the message transmission;

- Cluster head maintains a table of nodes connected to it as well as maintains some details about that node in the table;

- The wireless nodes are placed in the 2-dimensional Euclidean plane.

In Fast Clustering process, as the network setup phase starts initially all the nodes that lie in the range of the sink are directly connected to the sink. Out of these connected nodes the farthest layer of the nodes are selected for the further construction of the hierarchical network. Hence in this way the number of nodes connected or initial network construction time is minimized hence establishing a Faster Clustering process.

In the previous strategy, the whole load lies on the nearest nodes to the sink, hence using Fast Clustering we regulate the load evenly from the first layer to farthest possible layer which saves the nearest to sink layers energy in the initial time of the networks lifetime. As the power of the nodes of the current farthest layer that has been chosen for creating the hierarchical backbone decreases we move the Cluster Head role to the layers towards the sink hence this helps to maintain a balance between the nearest layer to the sink and the farthest layer with direct contact to sink as well as decreasing the network setup time hence justifying Fast Clustering.

Energy is not only imbalanced by just continuing the Cluster Head role of a node for a longer duration but also by un-even management of the number of member nodes between different cluster heads which in-turn results in a higher degree of one cluster head than the other. This un-even distribution is also addressed to in the Fast Clustering Strategy.

This issue is addressed in fast clustering by limiting the degree of the cluster head to a maximum value that can be assigned by the user based to the interest of the application and the need of the load-to-battery constraint. There are multiple cases to be considered in this regard as follows: Let $\mathrm{k}$ be the maximum degree of node permitted by the user for a node $\mathrm{x}$,

1. If $\mathrm{k}$ for a node $\mathrm{x}$ is more than number of nodes in vicinity to be added to the network then all the nodes to be assigned are added as member nodes to $\mathrm{x}$.

2. If $\mathrm{k}$ for a node is less than the available nodes to be assigned then, multiple nodes are selected as cluster heads to fulfil the demand, i.e. till all nodes are not assigned to some cluster head by keeping the degree constraint $\mathrm{k}$, new cluster heads are appointed.

In this way in Fast Clustering both uneven distribution of the degree of nodes, i.e. load as well as the imbalance in the usage of the resources of the nodes is tackled and the balance of resources is maintained.

Figure 1 shows proposed algorithm. 


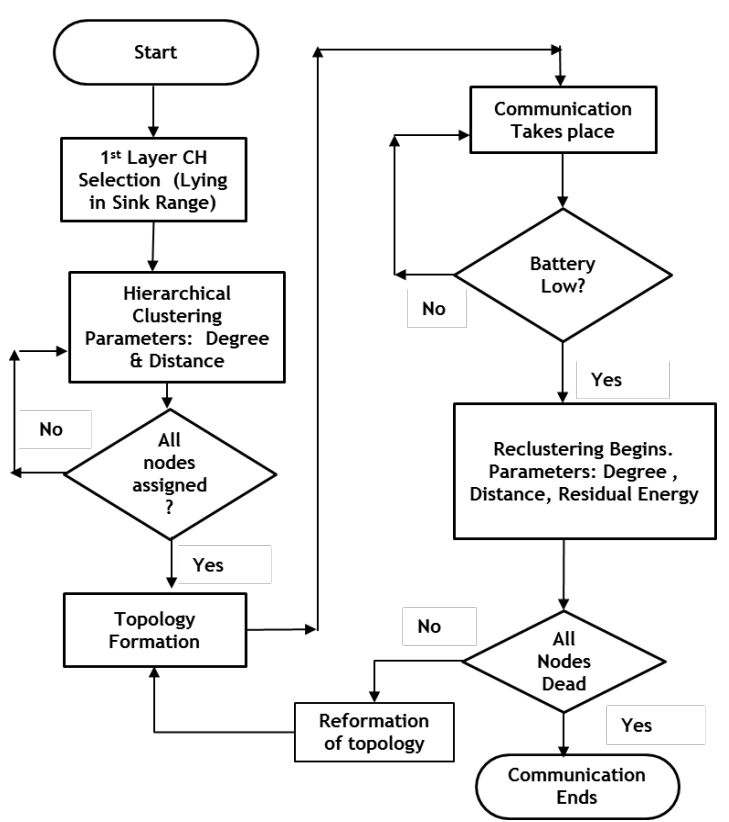

Figure 1: Proposed Algorithm

\section{Results}

Network Lifetime is the time interval from the start of operation of the sensor network until the death of the last alive node, i.e. when all energy in the network will be consumed. Here, network lifetime is defined as the time interval when the whole energy in the network will be consumed.

The Average Energy Consumption (AEC) is calculated across the entire network. It measures the average difference between the initial energy and the final energy of each node.

Let $\mathrm{Ei}=$ the initial energy of a node, $\mathrm{Ef}=$ the final energy of a node and $\mathrm{N}=$ number of nodes in the simulation.

$\mathrm{AEC}=\frac{\sum_{\mathrm{k}=1}^{\mathrm{S}}[\mathrm{Eik}-\mathrm{Efk}]}{\mathrm{N}}$

Table 2 shows various values of Network Lifetime corresponding to different sets of ranges and number of nodes. It has been observed that the Network Lifetime increases with decrease in Range of nodes and also by increasing number of nodes in the network. As such Lowest Range value of $30 \mathrm{~m}$ gives highest energy available to network after running for the specific time period.

The Table 3 shows various values of Average Energy Consumption corresponding to different sets of ranges and number of nodes.

\begin{tabular}{|c|c|c|c|c|c|c|}
\hline \multirow{2}{*}{$\begin{array}{c}\text { Number of } \\
\text { Nodes }\end{array}$} & \multicolumn{7}{|c|}{ Range in meters } \\
\cline { 2 - 7 } & 30 & 40 & 50 & 60 & 70 & 80 \\
\hline 50 & & 4252.89 & 3616.64 & 3257.03 & 2119.09 & 1786.6 \\
\hline 60 & 656529.84 & 3963.71 & 2994.96 & 2377.43 & 2350.37 & 1616.85 \\
\hline 70 & 555569.82 & 4476.46 & 3435.43 & 2209.05 & 2343.46 & 2058.16 \\
\hline 80 & 525217.09 & 3686.38 & 3318.39 & 2684.1 & 2298.19 & 1829.36 \\
\hline 90 & 6081.93 & 4640.15 & 3245.44 & 2674.23 & 2123.7 & 1748.3 \\
\hline 100 & 717100.69 & 5679.16 & 3424.14 & 2791.03 & 1937.56 & 1780.85 \\
\hline
\end{tabular}

Table 2: Network Lifetime (Seconds) Corresponding to Various Ranges.

\begin{tabular}{|c|c|c|c|c|c|c|c|}
\hline \multicolumn{2}{|c|}{ Number of Nodes } & 50 & 60 & 70 & 80 & 90 & 100 \\
\hline \multirow{4}{*}{ Range in meters } & 30 & & 11.79 & 14.19 & 17.05 & 15.94 & 14.78 \\
\cline { 2 - 8 } & 40 & 13.40 & 15.38 & 15.41 & 19.26 & 18.74 & 18.48 \\
\cline { 2 - 8 } & 50 & 13.54 & 18.36 & 15.42 & 19.98 & 18.57 & 23.07 \\
\cline { 2 - 8 } & 60 & 14.43 & 21.45 & 23.08 & 19.74 & 22.81 & 24.72 \\
\cline { 2 - 8 } & 70 & 18.40 & 16.59 & 17.49 & 22.19 & 20.24 & 26.32 \\
\cline { 2 - 8 } & 80 & 15.11 & 16.69 & 15.06 & 19.13 & 21.16 & 24.14 \\
\hline
\end{tabular}

Table 3: Average Energy Consumption (Joules) Corresponding to Various Ranges 


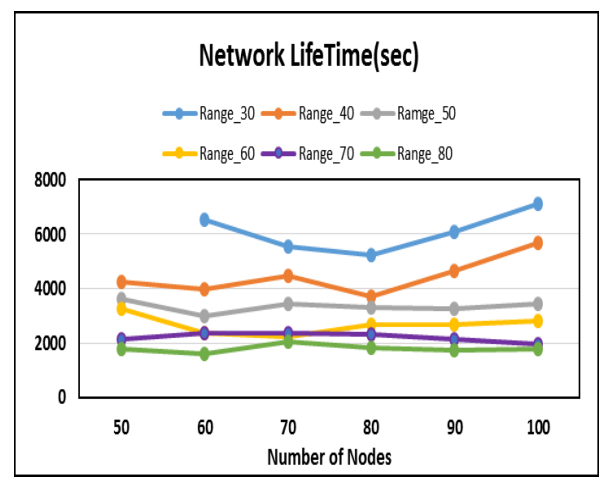

Figure 2: Effect of Ranges on Network Lifetime

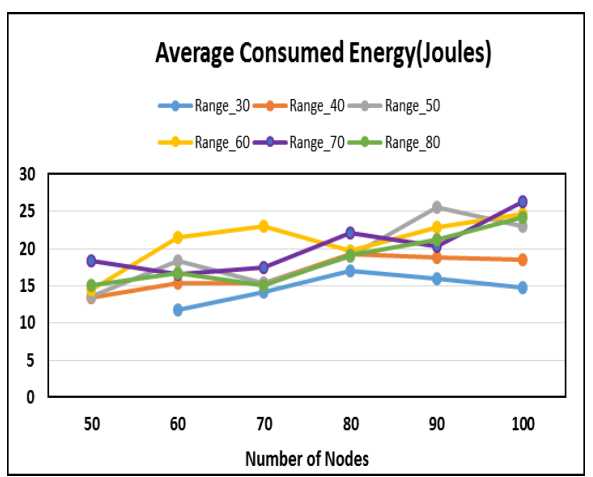

Figure 3: Effect of Ranges over Average Consumed Energy

It has been observed that more energy is consumed by network if number of nodes increases or range increases. As such the network consumed the least energy for range value of $30 \mathrm{~m}$ which tallies with the highest network life time.

Table 4 shows the theoretical comparison between MST, SOS, UDG and the proposed algorithm based on various factors.

\begin{tabular}{|c|c|c|c|c|}
\hline Parameter & $\begin{array}{c}\text { Minimum Spanning } \\
\text { Tree }\end{array}$ & Self - Organizing System & Unit Disk Graph & Proposed Algorithm \\
\hline Network Type & Static & Both Static and Dynamic & Static & Static \\
\hline Number of Links & Least & Interme-diate & Most & $\begin{array}{c}\text { Equal to number of } \\
\text { nodes }\end{array}$ \\
\hline Longevity of CH Role & $\begin{array}{c}\text { Relinqui-shes role after } \\
\text { certain threshold }\end{array}$ & $\begin{array}{c}\text { Sustains role by reducing } \\
\text { energy }\end{array}$ & $\begin{array}{c}\text { Relinquis-hes role } \\
\text { after certain threshold }\end{array}$ & $\begin{array}{c}\text { Relinquis-hes role after } \\
\text { certain threshold }\end{array}$ \\
\hline Flooding & Least & Interme-diate & Most & Directed Flooding \\
\hline Network Reconfigur-ation & Difficult & Relatively Easy & Easy & Easy \\
\hline Localization & Not much & Available & Available & Available \\
\hline Need of Control Message & Required & Not Required & Not Required & Not Required \\
\hline
\end{tabular}

Table 4: Comparison among MST, SOS, UDG and Proposed Algorithm.

\section{Conclusion}

The lifetime of a wireless sensor network is one of the most important parameters in study of WSNs. Since the resources available for a WSN are limited and continuously depleting, it is important to manage these resources efficiently. The hierarchical Clustering forming layers of $\mathrm{CH}$ and user-defined degree of each $\mathrm{CH}$ helps in load balancing and conserving energy reducing effect of network segmentation. This results in concentrated or Directed Flooding in network which forms the optimal route for nodes to communicate with sink.

\section{References}

[1] Guadalupe Olascuaga-Cabera, Ernesto Lopez-Mellado, Andres Mendez-Vazquez, and Felix Francisco RamosCorchado, "A Self-Organization Algorithm for Robust Networking of Wireless Devices", IEEE SENSORS JOURNAL, Vol 11, No. 3, March 2011 pp. 771-780

[2] Kuhn, F., Wattenhofer, R., \& Zollinger, A. (2008). Ad hoc networks beyond unit disk graphs. Wireless Networks, 14(5), pp. 715-729.

[3] Clark, B. N., Colbourn, C. J., \& Johnson, D. S., “Unit disk graphs”. Discrete mathematics, 86(1-3), pp. 165-177. 